OPEN ACCESS

Edited by:

Maria L. Pappas,

Democritus University of Thrace,

Greece

Reviewed by:

Elly Poretsky,

University of California, San Diego,

United States

Sybille B. Unsicker,

Max Planck Institute for Chemical

Ecology, Germany

*Correspondence:

Charles J. Mason

charles.mason@usda.gov

${ }^{\dagger}$ Present addresses: Charles J. Mason,

Tropical Crop and Commodity

Protection Research Unit,

Daniel K Inouye U.S. Pacific Basin

Agricultural Research Center,

Agricultural Research Service, USDA, Hilo, HI, United States

Swayamjit Ray,

Department of Plant Pathology and

Plant-Microbe Biology, Cornell

University, Ithaca, NY, United States

Elizabeth Davidson-Lowe,

U.S. Vegetable Laboratory,

Agricultural Research Service, United

States Department of Agriculture,

Charleston, SC, United States

Specialty section:

This article was submitted to

Chemical Ecology,

a section of the journal

Frontiers in Ecology and Evolution

Received: 27 December 2021

Accepted: 27 January 2022

Published: 25 February 2022

Citation:

Mason CJ, Ray S,

Davidson-Lowe E, Ali J, Luthe DS and

Felton G (2022) Plant Nutrition

Influences Resistant Maize Defense Responses to the Fall Armyworm

(Spodoptera frugiperda).

Front. Ecol. Evol. 10:844274. doi: 10.3389/fevo.2022.844274

\section{Plant Nutrition Influences Resistant Maize Defense Responses to the Fall Armyworm (Spodoptera frugiperda)}

\author{
Charles J. Mason ${ }^{1 * t}$, Swayamjit Ray ${ }^{1+}$, Elizabeth Davidson-Lowe ${ }^{1 \dagger}$, Jared Ali", \\ Dawn S. Luthe ${ }^{2}$ and Gary Felton ${ }^{1}$ \\ 1 Department of Entomology, The Pennsylvania State University, University Park, PA, United States, ${ }^{2}$ Department of Plant \\ Sciences, The Pennsylvania State University, University Park, PA, United States
}

Plants are often confronted by different groups of herbivores, which threaten their growth and reproduction. However, they are capable of mounting defenses against would-be attackers which may be heightened upon attack. Resistance to insects often varies among plant species, with different genotypes exhibiting unique patterns of chemical and physical defenses. Within this framework, plant access to nutrients may be critical for maximal functioning of resistance mechanisms and are likely to differ among plant genotypes. In this study, we aimed to test the hypothesis that access to nutrition would alter the expression of plant resistance to insects and alter insect performance in a manner consistent with fertilization regime. We used two maize (Zea mays) genotypes possessing different levels of resistance and the fall armyworm (Spodoptera frugiperda) as model systems. Plants were subjected to three fertilization regimes prior to assessing insect-mediated responses. Upon reaching V4 stage, maize plants were separated into two groups, one of which was infested with fall armyworm larvae to induce plant defenses. Plant tissue was collected and used in insect bioassays and to measure the expression of defense-related genes and proteins. Insect performance differed between the two plant genotypes substantially. For each genotype, fertilization altered larval performance, where lower fertilization rates hindered larval growth. Induction of plant defenses by prior herbivory substantially reduced naïve fall armyworm growth in both genotypes. The effects between fertilization and induced defenses were complex, with low fertilization reducing induced defenses in the resistant maize. Gene and protein expression patterns differed between the genotypes, with herbivory often increasing expression, but differing between fertilization levels. The soluble protein concentrations did not change across fertilization levels but was higher in the susceptible maize genotype. These results demonstrate the malleability of plant defenses and the cascading effects of plant nutrition on insect herbivory.

Keywords: herbivory, nitrogen, plant nutrition, plant defense, Lepidoptera

\section{INTRODUCTION}

Plants are often confronted by several simultaneous challenges, and the ability to partition resources adequately and efficiently is needed to ward against threats and balance productivity (Coley et al., 1985; Herms and Mattson, 1992; Stamp, 2003). As plants persist in heterogeneous environments, a central aspect of plant success against herbivores is balancing defense allocation with resource 
availability (Coley et al., 1985; Endara and Coley, 2011). Defenses against herbivores can include multiple components (Agrawal and Fishbein, 2006; Moles et al., 2013), each of which may be drawn from different resource pools (Gershenzon, 1994; Steppuhn and Baldwin, 2008). In general, plant defenses are not necessarily static, and can be heightened or enhanced by herbivory. Inducible defenses are strategies employed by plants that increase at the onset of herbivore pressure which minimize resources costs to the plant when they are not needed (Karban, 2011; Guo et al., 2018). Evidence of tradeoffs between resource variation and mobilization of inducible defenses has been supported in some instances (Agrawal et al., 2015; Burghardt, 2016), but not in others (Hale et al., 2005; Walls et al., 2005). A combination of the plant perceiving the specific herbivore (Ali and Agrawal, 2012) as well as selection of particular plant genotype(s) (Hahn and Maron, 2016) are contributors to these patterns.

The relationships between plants and insects in the context of resource partitioning and defenses is not unidirectional. Insects encounter heterogenous nutritional landscapes (Behmer, 2009), arising from species or genotypic traits associated with plant nutrient uptake ability or efficiency (Abbas et al., 2011; Wilson et al., 2019), as well as host-plant access to nutrients in the soil (Lavoie and Oberhauser, 2004; Prudic et al., 2005; Rashid et al., 2017). Insect performance metrics are commonly limited by the nutritional components of their diet (Awmack and Leather, 2002; Behmer, 2009), and nutrient variation can alter herbivore performance and population dynamics (Chen et al., 2010; Wetzel et al., 2016). Plant defenses can compound variability in diet quality (Hay et al., 1994; Behmer et al., 2002; Couture et al., 2016), and adversely impact the ability of herbivores to acquire nutritional resources through physical barriers (Phillips and Croteau, 1999; Sethi et al., 2008; Tian et al., 2012), by disrupting digestive processes (Barbehenn and Constabel, 2011; Zhu-Salzman and Zeng, 2015) or by degrading insect gut lining (Mohan et al., 2006; Mason et al., 2019).

Balancing the risk of herbivory with the ability to compete with other individuals is integral for plant success (Hambäck and Beckerman, 2003; Züst and Agrawal, 2017; de Vries et al., 2019). Over time, these processes can shape plant traits pertinent to natural and agricultural settings (Agrawal, 2007; Mertens et al., 2021). Since traits that confer resistance to insects are often desirable in agriculture (Mitchell et al., 2016), understanding how resource variation alters plant-herbivore interactions is important to determine if comparable levels of resistance are manifested in changing environments. Moreover, understanding the role that plant species or genotype play in maximizing resource use and inducible defenses will be critical for optimization of pest management strategies in dynamic environments.

The principal aim of our study was to determine how variation in resource availability influences antiherbivore defenses and subsequent herbivore performance. We sought to determine how nutrient access influences mobilization of rapid $(<24 \mathrm{~h})$ inducible defenses. We used plants with differing levels of anti-herbivore defenses to determine how plant genotype and nutrient availability contribute to variation in induced plantdefense responses.

To accomplish our objectives, we used two maize (Zea mays) genotypes exhibiting different levels of resistance to insect herbivory. Mp708 expresses a highly inducible, insecticidal cysteine protease (Mir1-CP) that impacts herbivores possessing diverse feeding strategies (Pechan et al., 2002; Mohan et al., 2006; Gill et al., 2011; Louis et al., 2015; Castano-Duque et al., 2017). Tx601, a parental line of Mp708 (Williams et al., 1990), is more palatable to defoliators than the resistant genotype. Nonetheless, Tx601 also possesses some defenses, such as trichomes, chitinases, ribosome inactivating proteins, and protease inhibitors that are induced upon attack and have negative impacts on folivores (Chuang et al., 2014; Mason et al., 2019; Han et al., 2021). Fall armyworm (Spodoptera frugiperda) was chosen as a model herbivore in our experiments. Fall armyworm has emerged as an important global pest across tropical and temperate regions around the world (Early et al., 2018; De Groote et al., 2020). Although fall armyworm is a highly polyphagous pest, it exhibits preference toward cereal crops (Montezano et al., 2018). Fall armyworm performance on Mp708 and Tx601 are well-documented (Pechan et al., 2002; Chuang et al., 2014), and maize defenses are known to alter digestive processes and induce septicemia (Fescemyer et al., 2013; Mason et al., 2019; Han et al., 2021). Because of the principal defenses that Mp708 and Tx601 weaponize are protein-based, we hypothesized that their expression may be limited by nutrient access. We also anticipated that since Mp708 has higher levels of resistance, susceptibility may be increased under nutrient stress. However, we also considered that when nutrients are limited, there may be cascading nutritional deficiencies onto fall armyworm, nullifying reduced defenses.

\section{MATERIALS AND METHODS}

\section{Plant and Insect Sources}

Maize lines Tx601 and Mp708 were selected for their respective susceptibility and resistance to different insects (Williams et al., 1990; Pechan et al., 2002; Louis et al., 2015), and seeds for both genotypes were kindly provided by Dr. Paul Williams. Fall armyworm were initially obtained from Benzon Research Inc. (Carlisle, PA, United States) and maintained in the laboratory. Two separate batches of fall armyworm larvae were reared from eggs to provide defoliator and bioassay insects.

\section{Plant Growing Conditions and Nutrient Regimes}

Maize seeds were germinated on moist paper towels under $100 \%$ relative humidity $(\mathrm{RH})$ at $28^{\circ} \mathrm{C}$ for $24 \mathrm{~h}$ prior to planting into substrate media. Seventy seeds of each genotype were transferred directly into Turface MVP Calcined Clay (PROFILE Products LLC., Buffalo Grove, IL., United States) in $10 \mathrm{~cm}$ square pots and maintained in a greenhouse at $27^{\circ} \mathrm{C}, 50-60 \% \mathrm{RH}, 14$-h-light/10h-dark photoperiod. Plants were watered daily until we randomly assigned plants into different fertilization regimes. 
Fertilization commenced 11 days post-planting and included three distinct regimes. Plants were randomly selected from the initial pool and 20 were assigned to each treatment. Fertilization was performed using water-soluble 20-10-20 Peters Professional fertilizer (JR Peters, Inc., Allentown, PA, United States) dissolved in State College, PA, United States tap water. Concentrations for low, medium (recommended), and high rates were: $1.29 \mathrm{~g} \mathrm{~L}^{-1}$ $2.58 \mathrm{~g} \mathrm{~L}^{-1}$, and $5.16 \mathrm{~g} \mathrm{~L}^{-1}$, respectively. One hundred $\mathrm{mL}$ of fertilization mixture was applied to each treatment group twice weekly 3-4 days apart, for a total of 8 instances of fertilizer application for the experiment's duration. Plants were watered to saturation on days they were not receiving fertilization. Fertilization treatments occurred until the onset of the V4 stage, 25 days following the first fertilization treatment.

\section{Defense Elicitation and Tissue Collection}

Eight plants of each genotype and fertilization treatment were infested with fall armyworm larvae in order to elicit defense responses the day following the last fertilization treatment. A single newly molted fifth instar fall armyworm larva was inserted into the whorl of plants assigned to infestation treatments. All control and infested plants were enclosed in individual fine mesh cages. Insects fed for $24 \mathrm{~h}$ before collection of foliage. Three plants assigned infestation treatments received no damage and were discarded from the experiment. Leaf tissues for bioassays and defense quantification were collected around the site of insect feeding within the whorl. Tissues from undamaged plants were collected from a comparable area of the plant. A portion of the tissue was flash frozen in liquid $\mathrm{N}_{2}$ and stored at $-80^{\circ} \mathrm{C}$ until we performed the biochemical analyses. The remaining leaf tissues were pooled within a treatment, cut into small pieces, and distributed equally among bioassay insects.

\section{Larval Bioassays}

Larval bioassays were performed in $22.5 \mathrm{~mL}$ diet cups containing $5-10 \mathrm{~mL}$ of $1.5 \%$ agar in a $22^{\circ} \mathrm{C}$ room under ambient light. Newly molted, laboratory-reared $3^{r d}$ instar fall armyworm were weighed and randomly assigned one of the nutrient-genotype-defense combinations (starting $n=27$ ). Leaves were distributed to ensure larvae did not run out of food at the beginning of the bioassay period. Bioassay larvae which did not exhibit any signs of feeding were discarded from the study, with final bioassay sample sizes ranging between 24 and 27 insects for each treatment. Bioassays were conducted for 6 days.

\section{Characterization of Inducible and Constitutive Defenses}

RNA was extracted as previously described (Ray et al., 2016). Approximately 100mg of tissue was homogenized in liquid nitrogen, and RNA was extracted with $1 \mathrm{~mL}$ of Trizol (Thermo-Fisher Scientific, Waltham, MA) followed by lithium chloride precipitation to remove contaminating DNA. Complementary DNA (cDNA) was synthesized from RNA using the High Capacity cDNA reverse transcription kit (Applied Biosystems, Foster City, CA) following manufacturer's instructions. Quantitative RT-PCR analyses were conducted on a QuantStudio 3 PCR system (Thermo Fisher, United States). Reactions were performed with SYBR Green PCR Mix (Applied Biosystems) using ubiquitin as the endogenous gene and responses were calculated using the $-2^{\Delta \Delta C T}$ method (Livak and Schmittgen, 2001) and ThermoFisher software, with low nutrient-no defoliation serving as the reference control for each genotype. In the susceptible maize genotype Tx601, ribosome inactivating protein (Rip2), which is downstream of jasmonic acid induced defense pathway was shown to lower caterpillar performance on plants (Chuang et al., 2014). However, in the resistant genotype Mp708, jasmonic acid was constitutively upregulated and the cysteine protease Mir1-CP was the primary defense related protein that was involved in insect resistance (Pechan et al., 2000; Shivaji et al., 2010; Fescemyer et al., 2013). Therefore, these two genes were measured as markers for caterpillar induced defenses in quantitative real time PCRs in Tx601 and Mp708, respectively. We also measured the transcript abundance of a pathogenesis related gene (Pr5) which has been shown to be downstream of salicylic acid defense pathway in maize and is usually not induced by caterpillar herbivory in maize (Ray et al., 2016). Gene targets and primer sequences are provided in Table 1.

\section{Immunoblot Methods}

Total protein from maize leaves were extracted from $100 \mathrm{mg}$ of tissue as described previously (Chuang et al., 2014). Briefly, plant tissues were homogenized and boiled in $1 \times$ Laemli sample buffer for $10 \mathrm{~min}$, followed by centrifuging them for $10 \mathrm{~min}$ at $14,000 \mathrm{~g}$. The supernatant was collected and protein content was measured using Non-interfering protein quantification kit (G-Biosciences, St. Louis, MO, United States). A total of $50 \mu \mathrm{g}$ of protein pool with equal amount of protein from each of six biological replicates for each treatment were loaded on to $10 \%$ polyacrylamide gel and ran for $90 \mathrm{~min}$ at 100 constant voltage. Proteins were transferred to nitrocellulose membrane using a wet transfer system overnight at $4^{\circ} \mathrm{C}$, following which they were blocked for an hour in 5\% milk and then incubated with 1:10,000 dilution of antibodies for RIP2 and Mir1-CP overnight at $4^{\circ} \mathrm{C}$ (Chuang et al., 2014; Louis et al., 2015). Polyclonal antibodies that were generated in rabbit of RIP2 and Mir1-CP were procured from Dr. Rebecca Boston's laboratory (Bass et al., 2004) and commercially synthesized, respectively (GenScript, NJ, United States). Secondary antibodies conjugated to horse radish peroxidase (Sigma Aldrich, United States) were used to bind to the primary antibodies in the ratio $1: 10,000$ for an hour at room temperature. The blots were then washed and visualized using Super Signal Femto substrate as per manufacturer protocol (Thermo Fisher Scientific, United States). The blots were stained with Ponceau $S$ to show equal protein loading and transfer in each well.

\section{Quantification of Foliar Protein}

To determine how nutrient treatments altered foliar protein concentrations, we evaluated bulk protein concentrations in fresh foliage. One hundred fifty-200 mg of frozen tissue was quantified and ground to a fine powder in liquid nitrogen. Soluble protein was extracted in $1 \mathrm{~mL}$ of Tris buffer $(\mathrm{pH} 6.8)$ 
TABLE 1 | Primers used in this study for rt-PCR.

\begin{tabular}{|c|c|c|c|c|}
\hline Gene and Transcript & Forward primer $\left(5^{\prime}-3^{\prime}\right)$ & Reverse primer $\left(5^{\prime}-3^{\prime}\right)$ & Transcript Accession & Primer Citation \\
\hline Maize ubiquitin & CAGGTGGGGTATTCTTGGTG & ATGTTCGGGTGGAAAACCTT & GRMZM2G102471 & This study \\
\hline Maize protease inhibitor (mpi) & GCGGATTATCGCCCTAACC & CGTCTGGGCGACGATGTC & X78988.2 & Louis et al., 2015 \\
\hline Ribosomal inactivating protein2 (rip2) & GAGATCCCCGACATGAAGGA & CTGCGCTGCTGCGTIT & M83926 & Chuang et al., 2014 \\
\hline $\begin{array}{l}\text { Maize insect resistance1 cysteine } \\
\text { protease (mir1-cp) }\end{array}$ & GATGGTCTTGTCGTGTTGAACTT & GCCACACCATAACGGATTAACTT & AF019145 & Louis et al., 2015 \\
\hline Pathogenesis-related gene5 (pr5) & TGCATGCATGGGCTAGTGAT & CGCACACAAATCCAGCTACG & U82201 & Morris et al., 1998 \\
\hline
\end{tabular}

Ubiquitin was utilized as an endogenous control.

containing 3\% SDS, 10\% glycerol, and 5\% 2-mercaptaethanol by incubating at $100^{\circ} \mathrm{C}$ for $10 \mathrm{~min}$. Proteins were quantified on a SpectraMax spectrophotometer (Molecular Devices, Sunnyvale, CA, United States) with a non-interfering protein quantification kit, according to the manufacturer's instructions (G-Biosciences, St. Louis, MO, United States).

\section{Statistical Analyses}

Analyses were performed with R (R Core Team, 2020) implemented in RStudio v. 4.1.1 (RStudio Team, 2015). Before performing analyses, data were evaluated for normality and homoscedasticity of variances and data transformations were performed as necessary. Larval bioassays were analyzed using an analysis of covariance (ANCOVA), with final body mass as the response variable, nutritional status and defoliation treatment as categorical fixed effects, and initial body mass as a random effect. We analyzed each genotype with separate ANCOVAs. Quantitative RT-PCR data were $\log _{10}$ transformed and analyzed using an ANOVA with nutritional status and defoliation treatment as fixed effects. Foliar protein concentrations were analyzed with ANOVA using plant genotype and nutritional status as fixed effects. Post hoc analyses for ANCOVAs and ANOVAs were performed using a Tukey HSD correction implemented in the R package "agricolae" (de Mendiburu, 2014).

\section{RESULTS}

\section{Fall Armyworm Growth Responses}

Fall armyworm growth responses followed our predictions with the two maize genotypes we used for our study (Figure 1). Fall armyworm exhibited $1.5 \times$ greater body mass accumulation on Tx601 plants maintained under a moderate nutritional regimen and no herbivory treatment compared to Mp708 (Supplementary Figure 1; $t_{49}=3.86$; $p<0.001)$. Both fertilization regimes and herbivory altered fall armyworm performance. For Tx601, prior herbivory substantially reduced $(\sim 25 \%)$ fall armyworm larval body mass (Table 2A; $F_{1,143}=20.6 ; p<0.001$ ), particularly among the low and medium fertilization treatments. Likewise, fertilization treatments altered fall armyworm performance $\left(F_{2,143}=4.49\right.$; $p=0.013$ ), but these effects were less pronounced. While there was no statistically significant interaction between fertilization and herbivory $\left(F_{2,143}=1.03 ; p=0.359\right)$, pairwise comparisons indicated fall armyworm had increased body mass on the high nutritional treatment compared to the medium and low treatments. This was evident among the plants receiving herbivory, where the high nutrient treatment resulted in caterpillars $1.5 \times$ larger than the other two fertilization treatments. As with Tx601, larvae consuming Mp708 had negative growth responses to herbivory (Figure $\mathbf{1}$ and Table 2B). While statistically significant $\left(F_{2,144}=3.24 ; p=0.042\right)$, the impact of fertilization was more complicated since we observed a significant interaction between prior herbivory and the fertilization treatments $\left(F_{2,144}=10.6 ; p<0.001\right)$. When consuming undamaged tissue, larvae performed poorly on the low fertilization treatment, exhibiting 50\% less body mass accumulation compared to the medium and high treatments. Low fertilization inhibited the induction of defenses to herbivory, where damaged and undamaged tissues did not differ under this regimen. The highest fertilization treatment exhibited reduction in herbivory but was not different from the lowest treatment. The medium fertilization treatment exhibited high levels of induction both in terms of how the larvae faired on the tissue and how it compared to the undamaged material.

\section{Plant Biochemical Responses to Fertilization and Herbivory Treatments}

We observed variable patterns in how defense-related gene expression (Figure 2 and Table 3 ) and proteins (Figure 3) responded to fertilization and herbivory between the two genotypes. Tx601 expression of maize protease inhibitor (mpi) and ribosome inactivating protein2 (rip2) transcripts followed identical trends (Figure 2). In both cases, there was no effect of fertilization on the expression (Table 3) but exhibited strong $(10 \times)$ increases in response to herbivory $(p<0.001)$. Immunoblot detection of RIP2 in Tx601 foliage followed identical trends (Figure 3), in which no detection was observed in undamaged foliage with high expression occurring in damaged foliage. Defense responses in Mp708 were more nuanced with regards to fertilization than Tx601. Mp708 mpi expression likewise increased by $5-10 \times$ in response to herbivory $\left(F_{1,30}=118.4 ; \mathrm{p}<0.001\right)$, but we also observed a response to fertilization $\left(F_{2,30}=6.42 ; p=0.005\right)$. This effect was apparent in damaged tissues, where the highest fertilization treatment exhibited the lowest expression. Mp708 pathogen response 5 (pr5) expression was not affected by fertilization, but increased by $1.5-4 \times$ in response to herbivory $\left(F_{1,30}=9.40 ; p=0.005\right)$. Expression of Mp708 MIR1-CP (mir1cp) did not change during herbivory. The expression of 

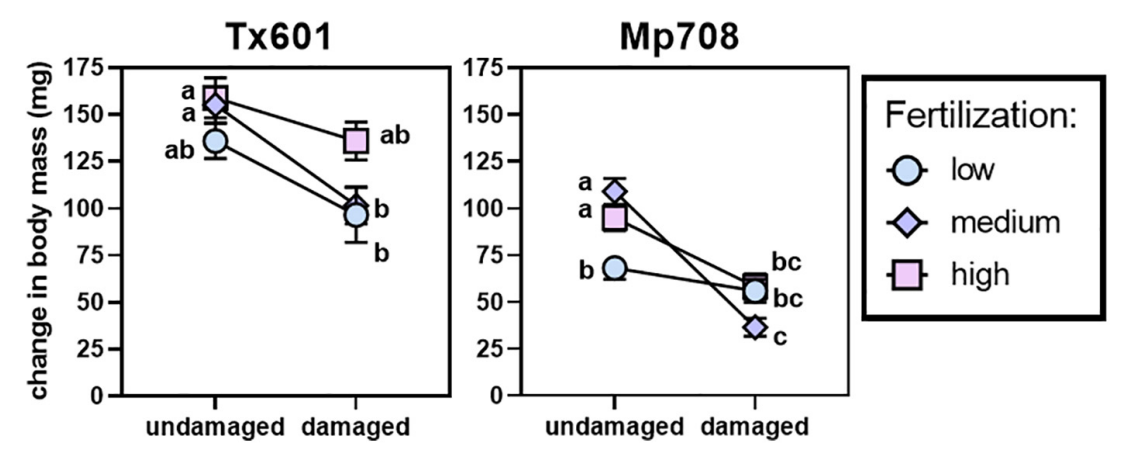

FIGURE 1 | Norm-reactive plot illustrating impacts of maize genotype, herbivory, and fertilization regime on fall armyworm growth. Symbols represent means with bars representing standard errors. Lines connect undamaged and damaged treatments. Different letters represent statistically significant responses within a genotype using a Holm correction $(p<0.05)$. Data were analyzed using final mass as response variable in an ANCOVA, and full statistics are present in Table 2.

mir1cp transcripts was discordant with the mobilization of the protein in the foliar tissues (Figure 3), as it responded to both herbivory and fertilization. MIR1-CP was not detected with immunoblotting in both undamaged and damaged Mp708 on the lowest fertilization treatment. In contrast, MIR1-CP accumulated in both medium and high fertilization treatments, with band intensity increasing upon herbivory.

\section{Soluble Protein Concentrations in Response to Genotype and Fertilization}

To determine how fertilization alters foliar quality, we evaluated soluble protein concentrations in the two genotypes (Figure 4). We observed differences in protein content between the two genotypes $\left(F_{1,25}=8.55 ; p=0.007\right)$, with $\mathrm{Tx601}$ having $\sim 1.5 \times$ higher concentrations compared to Mp708. However, we saw no overall effect of fertilization on the level of foliar protein $\left(F_{2,25}=0.25 ; p=0.781\right)$ and no interaction with plant genotype $\left(F_{2,25}=2.68 ; p=0.090\right)$.

\section{DISCUSSION}

Heterogenous nutritional environments can lead to cascading effects on plant and insect populations (Wetzel et al., 2016). Plant performance and growth are driven by nutrient availability, and nutrient deficiencies can have cascading effects on the mobilization of plant defenses and resistance to herbivores. Herbivores must fulfill their own nutritional requirements while often simultaneously contending with toxic plant compounds and, therefore, must strategize between nutrient acquisition and toxin avoidance or detoxification. Our study demonstrates that regulating nutrient access to maize plants has cascading effects on fall armyworm larvae via modulation of plant defenses. It is well-documented that plant genetics play a role in defense variability (Bruce, 2014) and, as anticipated, we observed differences between resistant and susceptible maize genotypes. Using two genotypes, we found that fertilization impacted herbivore performance and defense accumulation. In general, larvae performed worse on plants receiving lower fertilization treatments and overall larval performance was higher on the susceptible genotype (Tx601) compared to the resistant genotype (Mp708). While both genotypes increased defense concentrations that impaired larval growth, the strength of that effect differed by genotype and level of fertilization. Interestingly, while total protein content was higher in Tx601, we did not observe an effect due to fertilization treatment. Our results support prior work indicating that nutritional access is integral to mounting successful defenses, and can have mixed, cascading effects on herbivores.

Quantitative PCR results exhibited strong responses to induction, but the patterns among fertilization treatments were variable. Prior studies have shown a correlation between available soil nitrogen to phosphorous ratio to induction of plant defense genes (Murrell et al., 2019). For Tx601, expression of mpi and rip 2 were induced by herbivory, but fertilization had no effect on defense gene expression. Similarly, in Mp708, pr5 expression was higher after herbivore damage but was not affected by fertilization. However, expression of mirlcp was nutrient-dependent but did not differ between constitutive and induced plant tissues. These results suggest that prioritization of certain defenses in response to nutrient availability varies and

TABLE 2 | ANCOVA table analyzing effects of fertilization treatment, and herbivory on fall armyworm growth for the two maize genotypes.

\begin{tabular}{lccc}
\hline A. Tx601: & & & \\
\hline Effect: & Df & F-value & P-value \\
\hline fertilization & 2,143 & 4.49 & $\mathbf{0 . 0 1 3}$ \\
herbivory & 1,143 & 20.6 & $<\mathbf{0 . 0 0 1}$ \\
fertilization:herbivory & 2,143 & 6.83 & 0.359 \\
B. Mp708 & & & \\
\hline Effect: & Df & F-value & P-value \\
\hline fertilization & 2,144 & 3.24 & $\mathbf{0 . 0 4 2}$ \\
herbivory & 1,144 & 60.2 & $<\mathbf{0 . 0 0 1}$ \\
fertilization:herbivory & 2,144 & 10.6 & $<\mathbf{0 . 0 0 1}$
\end{tabular}

Final larval mass was used as response variable, with initial larval mass was used as the covariate. Bolded values denote statistically significant terms in the model. 

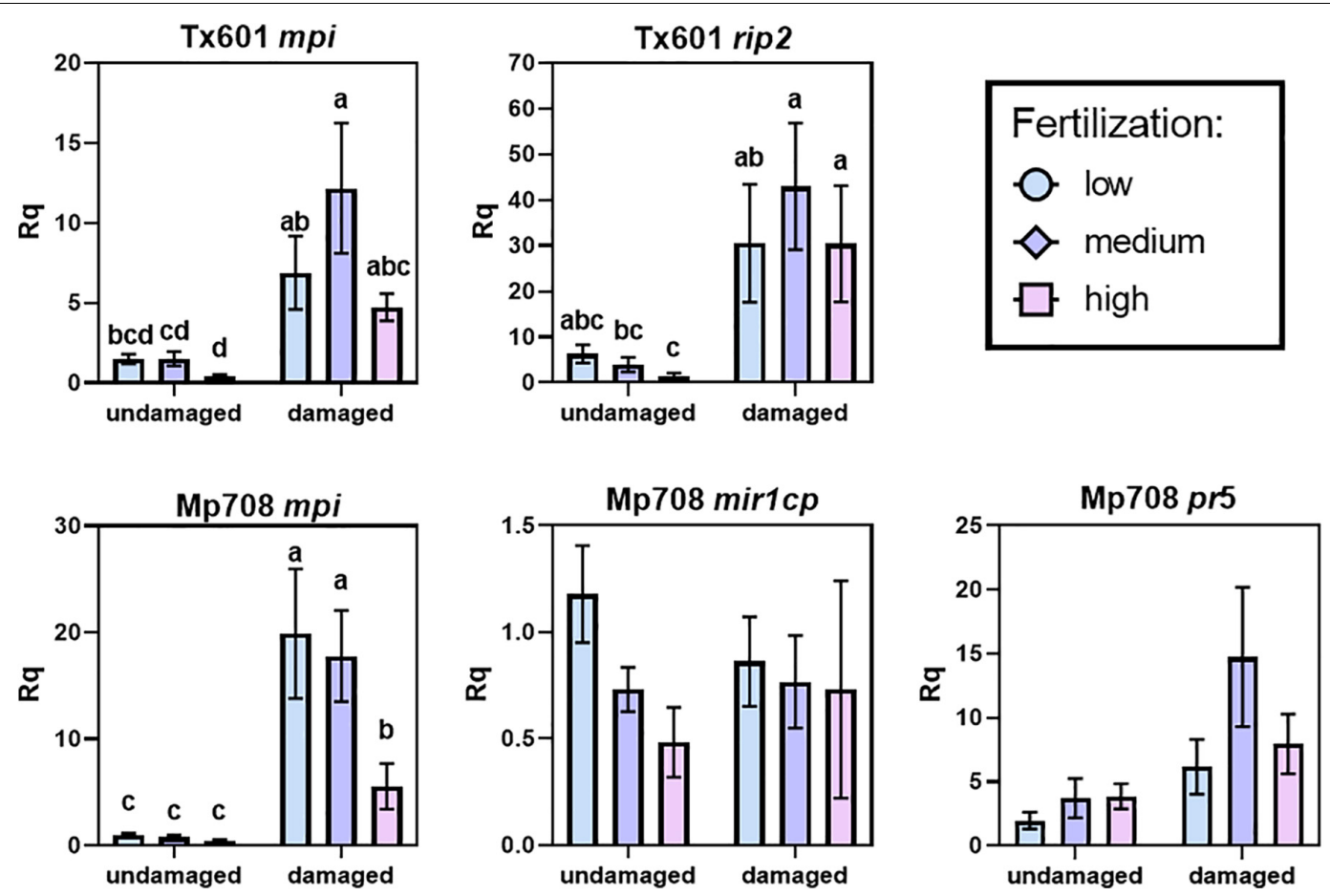

FIGURE 2 | Influence of herbivory and fertilization on the expression of defense-related genes in Tx601 and Mp708. Letters represent statistically significant pairwise comparisons within a transcript. Statistical analyses were conducted using $\log _{10}(y)$ transformed responses. Full statistics are presented in Table 3. rip2 - ribosome inactivating protein 2; mpi - maize protease inhibitor; mir1cp - Mir1 cysteine protease; pr5 - pathogen response protein 5.

that these genotypes may employ different strategies to manage nutrient and herbivore stress. While these data are certainly insightful, transcript levels are not always correlated to protein levels in cells. Therefore, we performed immunoblot assays to quantify some of the defense related proteins.

Immunoblots of defense proteins best explain the patterns we observed in our bioassay data. The immunoblot of defense protein RIP2 in the susceptible genotype Tx601 revealed higher accumulation of the protein when induced by caterpillar feeding. This suggests that although Tx601 is more susceptible to insect herbivory, its ability to mount a successful defense response is not contingent on soil nutrient availability. Tx601 RIP2 protein has previously been shown to be insecticidal (Chuang et al., 2014) and supports our bioassay results where we saw that regardless of fertilizer regimen, induced plants were more resistant to subsequent insect herbivory. In contrast, we found that accumulation of the defense protein MIR1-CP in the resistant genotype Mp708 was positively associated with nutrient availability. MIR1-CP accumulation was not observed in plants grown in soil with low nutrients. However, plants grown with medium and high fertilizer treatments accumulated higher levels of this defensive protein. Therefore, in Mp708 plants, maximization of this defense component is contingent on fertilization access. We can only speculate as to why fall armyworm performed poorly on low fertilization-constitutive Mp708. This genotype is known to constitutively exhibit higher levels of jasmonic acid (Shivaji et al., 2010), a key phytohormone in defense signaling as well in mediating abiotic stress responses
(Raza et al., 2021). Since the genome of Mp708 carries several QTLs that are involved in imparting resistance to insect herbivores (Castano-Duque and Luthe, 2018), it is possible that the regulation of fall armyworm growth on these plants was mediated through defenses that were not measured here (i.e., benzoxazinoids, lignin, other phenolics) (for example Tzin et al., 2017) or some other essential nutrients (i.e., micronutrients, digestible carbohydrates) (for example Apple et al., 2009).

Over the past several decades, many studies have aimed to identify how ecological constraints, such as nutrient availability affect plant defenses, but the patterns are not always clear. Documented effects of nutrient availability on rapid-inducible

TABLE 3 | ANOVA table of maize transcripts and their expression as affected by fertilization and herbivory.

\begin{tabular}{|c|c|c|c|c|c|c|c|}
\hline & & \multicolumn{2}{|c|}{$\underline{\text { Fertilization }}$} & \multicolumn{2}{|c|}{ Herbivory } & \multicolumn{2}{|c|}{ Fertilization:Herbivory } \\
\hline \multicolumn{2}{|c|}{$\begin{array}{l}\text { Genotype and } \\
\text { Transcript: }\end{array}$} & F-value & P-value & F-value & P-value & F-value & P-value \\
\hline \multirow[t]{2}{*}{ Tx601: } & mpi & 2.13 & 0.138 & 16.9 & $<0.001$ & 1.363 & 0.272 \\
\hline & rip2 & 1.05 & 0.364 & 29.3 & $<0.001$ & 2.08 & 0.143 \\
\hline \multirow[t]{3}{*}{ Mp708: } & mpi & 6.42 & 0.005 & 118.4 & $<0.001$ & 0.848 & 0.438 \\
\hline & mir1cp & 5.49 & 0.010 & 0.91 & 0.348 & 0.096 & 0.909 \\
\hline & pr5 & 1.27 & 0.296 & 9.40 & 0.005 & 0.166 & 0.848 \\
\hline
\end{tabular}

Values were log(y)-transformed prior to analysis. Bolded values indicate statistically significant effects. 
RIP2 in Tx601

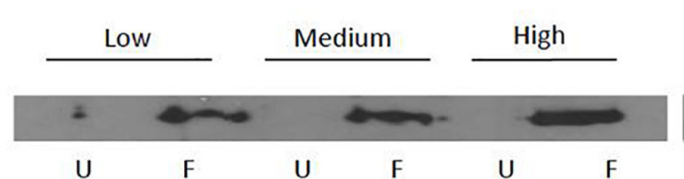

Ponceau S
Mir1-CP in Mp708

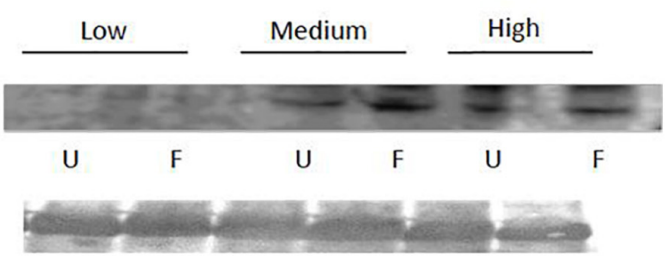

FIGURE 3 | Influence of herbivory and fertilization on the protein levels of RIP2 and MIR1-CP in Tx601 and Mp708, respectively. Immunoblot detection of defense related proteins RIP2 and MIR1-CP was performed in Tx601 and Mp708, respectively, for plants with low, medium and high regimens of fertilizer. Within each fertilizer treatments, plants were either left undamaged $(U)$ or fed with $5^{\text {th }}$ instar fall armyworm larva for $24 \mathrm{hrs}(\mathrm{F})$. Each well represents $50 \mu \mathrm{g}$ of total protein that was pooled from equal amounts of protein from six plants, equal loading and transfer is shown in Ponceau S stain of the blots.

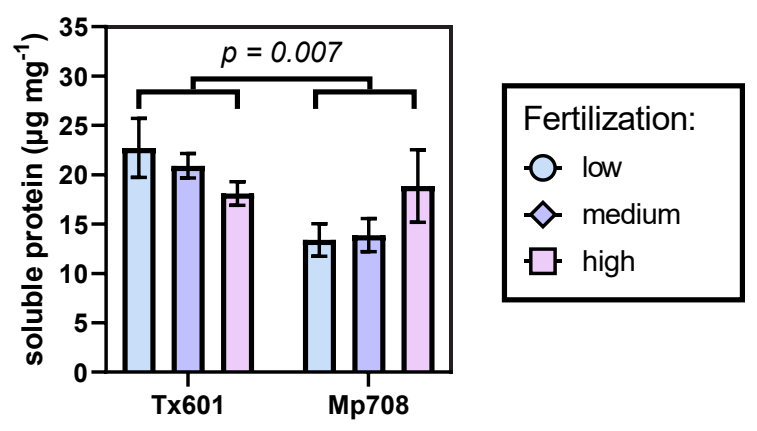

FIGURE 4 | Influence of fertilization treatment on soluble protein concentrations (fresh weight) in Tx601 and Mp708 foliage. Different concentrations of total proteins were observed between genotypes, but not within a fertilization regime.

defenses have ranged from positive, neutral to negative (Stout et al., 1998; Cipollini and Bergelson, 2001; Schmelz et al., 2003), making it difficult to explain or predict the outcomes of these biochemical interactions. For example, decreasing nutrients reduced the ability of rapeseed plants to induce defenses, but high nutrient availability restored induction capacity (Cipollini and Bergelson, 2001). Contradictory to our results, reducing nutrient availability in maize (cv. Delprim) led to an increase in the induction of defense-related plant terpenes and phytohormones (Schmelz et al., 2003). Similar results were found in cotton, where lower fertilization rates resulted in increased herbivoreinduced defenses (Chen et al., 2008). Many variables, such as fertilizer source, ratio, or composition, as well as type of damage (herbivory, elicitor, mechanical) make it difficult to draw comparisons between studies. However, as was evidenced in our study, genetic and environmental factors are both involved in allocation of resources toward induced defenses, highlighting the need for further evaluation of resistance traits across genotypes and under different environmental contexts to attain consistent and predictable outcomes.

We used a three-macronutrient fertilizer blend with higher concentrations of nitrogen and potassium compared to phosphorous. It is not certain to what extent each nutrient, or combination of nutrients, played a predominant roll in limiting defense induction. Other research has shown that limiting nitrogen is linked to suppression of nitrogen-rich proteins, such as those used for plant defenses (Lou and Baldwin, 2004). In our study, the low fertilization treatment suppressed constitutive and induced expression of MIR1-CP in the resistant genotype. However, nitrogen is also a limiting nutrient to herbivore growth and nitrogen availability is associated with fall armyworm performance and behavior. Fall armyworm typically perform better on and prefer plants that are grown in nitrogen-rich soils (Wiseman et al., 1973; Davidson-Lowe et al., 2021). Recent studies demonstrate that phosphorous plays a functional role in defense hormone signaling and is also involved in plant-microbe associations that confer resistance to herbivores and pathogens (Mustafa et al., 2016; Murrell et al., 2019; Chan et al., 2021). Additionally, potassium may have an inhibitory effect on fall armyworm growth and development (Singh et al., 2021), though the mechanisms are unclear. Future comparative studies that isolate and manipulate nutrients will shed light on nutrientspecific effects on plant defenses and help determine whether the benefits of nutrient access override defense constraints for herbivore host-plant selection. Our experiments were conducted in the greenhouse under controlled conditions, and therefore future field studies are needed to understand if the processes we observed here are consistent across habitats.

Interactions between nutrients, plant defenses and herbivores are undeniably complex. Including genotypic variability further complicates these relationships. Here we demonstrated that plant genotype and nutrient availability affect plant-herbivore interactions by differentially influencing the expression of plant defenses. This study provides insight on how these interactions may contribute to plant-herbivore community assemblage in natural and agricultural landscapes. Subsequent studies are needed to understand how these patterns translate to field situations, the plant tolerance of and recover from herbivory on defense responses, and how different nutritional components alter the defense cascades.

\section{DATA AVAILABILITY STATEMENT}

The original contributions presented in the study are included in the article/Supplementary Material, further inquiries can be directed to the corresponding author/s. 


\section{AUTHOR CONTRIBUTIONS}

CM and SR conceived of the experiment, designed the study, carried out the experiments, and analyzed the data. GF and JA contributed funding. CM and ED-L wrote the initial manuscript draft with other authors provided editorial assistance. All authors provided feedback on data interpretation.

\section{FUNDING}

This research was supported in part by an appointment to the Agricultural Research Service (ARS) Research Participation Program administered by the Oak Ridge Institute for Science and Education (ORISE) through an interagency agreement between the US Department of Energy (DOE) and the US Department of Agriculture (USDA). ORISE is managed by ORAU under DOE contract number DE-SC0014664.

\section{ACKNOWLEDGMENTS}

We thank W. Paul Williams for providing seed and Rebecca Boston for providing RIP2 polyclonal antibodies. We appreciate Ebony Murrell at The Land Institute, Kansas, United States, for

\section{REFERENCES}

Abbas, F., Morellet, N., Hewison, A. J. M., Merlet, J., Cargnelutti, B., Lourtet, B., et al. (2011). Landscape fragmentation generates spatial variation of diet composition and quality in a generalist herbivore. Oecologia 167, 401-411. doi: 10.1007/s00442-011-1994-0

Agrawal, A. A. (2007). Macroevolution of plant defense strategies. Trends Ecol. Evol. 22, 103-109. doi: 10.1016/j.tree.2006.10.012

Agrawal, A. A., and Fishbein, M. (2006). Plant defense syndromes. Ecology 87, 132-149.

Agrawal, A. A., Hastings, A. P., Bradburd, G. S., Woods, E. C., Züst, T., Harvey, J. A., et al. (2015). Evolution of plant growth and defense in a continental introduction. Am. Nat. 186, E1-E15. doi: 10.1086/68 1622

Ali, J. G., and Agrawal, A. A. (2012). Specialist versus generalist insect herbivores and plant defense. Trends Plant Sci. 17, 293-302. doi: 10.1016/j.tplants.2012.02. 006

Apple, J. L., Wink, M., Wills, S. E., and Bishop, J. G. (2009). Successional change in phosphorus stoichiometry explains the inverse relationship between herbivory and lupin density on Mount St. Helens. PLoS One 4:e7807. doi: 10.1371/journal. pone.0007807

Awmack, C. S., and Leather, S. R. (2002). Host plant quality and fecundity in herbivorous insects. Annu. Rev. Entomol. 47, 817-844. doi: 10.1146/annurev. ento.47.091201.145300

Barbehenn, R. V., and Constabel, C. (2011). Tannins in plant-herbivore interactions. Phytochemistry 72, 1551-1565. doi: 10.1016/j.phytochem.2011.01. 040

Bass, H. W., Krawetz, J. E., OBrian, G. R., Zinselmeier, C., Hebben, J. E., and Boston, R. S. (2004). Maize ribosome-inactivating proteins (RIPs) with distinct expression patterns have similar requirements for proenzyme activation. J. Exp. Bot. 55, 2219-2233. doi: 10.1093/jxb/erh243

Behmer, S. T. (2009). Insect herbivore nutrient regulation. Annu. Rev. Entomol. 54, 165-187. doi: 10.1146/annurev.ento.54.110807.090537

Behmer, S. T., Simpson, S. J., and Raubenheimer, D. (2002). Herbivore foraging in chemically heterogeneous environments: nutrients and secondary metabolites. Ecology 83, 2489-2501. doi: 10.1890/0012-9658(2006)87[2236:ssotpo] 2.0.co;2 her input into the experimental setup. Funding was provided by United States Department of Agriculture NIFA Postdoctoral Fellowship 2018-67012-27979 awarded to CM, US Department of Agriculture AFRI Grant 2017-67013-26596 awarded to GF, and Hatch Project Grant PEN04576. This research was supported in part by the U.S. Department of Agriculture, Agricultural Research Service. The findings and conclusions in this publication are those of the authors and should not be construed to represent any official USDA or U.S. Government determination or policy. Mention of trade names or commercial products in this publication is solely for the purpose of providing specific information and does not imply recommendation or endorsement by the U.S. Department of Agriculture. USDA is an equal opportunity provider and employer.

\section{SUPPLEMENTARY MATERIAL}

The Supplementary Material for this article can be found online at: https://www.frontiersin.org/articles/10.3389/fevo.2022. 844274/full\#supplementary-material

Supplementary Figure 1 | Comparison of fall armyworm growth feeding on Tx601 and Mp708 under medium nitrogen treatments without herbivory.

Bruce, T. J. A. (2014). Variation in plant responsiveness to defense elicitors caused by genotype and environment. Front. Plant Sci. 5:349. doi: 10.3389/fpls.2014. 00349

Burghardt, K. T. (2016). Nutrient supply alters goldenrod's induced response to herbivory. Funct. Ecol. 30, 1769-1778. doi: 10.1111/1365-2435.12681

Castano-Duque, L., and Luthe, D. S. (2018). Protein networks reveal organ-specific defense strategies in maize in response to an aboveground herbivore. Arthropod. Plant. Interact. 12, 147-175. doi: 10.1007/s11829-017-9562-0

Castano-Duque, L., Loades, K. W., Tooker, J. F., Brown, K. M., Williams, W. P., and Luthe, D. S. (2017). A maize inbred exhibits resistance against western corn rootwoorm, Diabrotica virgifera virgifera. J Chem Ecol. 43, 1109-1123. doi: 10.1007/s10886-017-0904-2

Chan, C., Liao, Y.-Y., and Chiou, T.-J. (2021). The impact of phosphorus on plant immunity. Plant Cell Physiol. 62, 582-589. doi: 10.1093/pcp/pcaa168

Chen, Y., Olson, D. M., and Ruberson, J. R. (2010). Effects of nitrogen fertilization on tritrophic interactions. Arthropod. Plant. Interact. 4, 81-94. doi: 10.1007/ s11829-010-9092-5

Chen, Y., Schmelz, E. A., Wäckers, F., and Ruberson, J. R. (2008). Cotton plant, Gossypium hirsutum L., defense in response to nitrogen fertilization. J. Chem. Ecol. 34, 1553-1564. doi: 10.1007/s10886-008-9560-x

Chuang, W. P., Herde, M., Ray, S., Castano-Duque, L., Howe, G. A., and Luthe, D. S. (2014). Caterpillar attack triggers accumulation of the toxic maize protein RIP2. New Phytol. 201, 928-939. doi: 10.1111/nph.12581

Cipollini, D. F., and Bergelson, J. (2001). Plant density and nutrient availability constrain constitutive and wound-induced expression of trypsin inhibitors in Brassica napus. J. Chem. Ecol. 27, 593-610. doi: 10.1023/A:1010384805014

Coley, P. D., Bryant, J. P., and Chapin, F. S. (1985). Resource availability and plant antiherbivore defense. Science 230, 895-899. doi: 10.1126/science.230.4728.895

Couture, J. J., Mason, C. J., Habeck, C. W., and Lindroth, R. L. (2016). Behavioral and morphological responses of an insect herbivore to low nutrient quality are inhibited by plant chemical defenses. Arthropod. Plant. Interact. 10, 341-349. doi: 10.1007/s11829-016-9439-7

Davidson-Lowe, E., Ray, S., Murrell, E., Kaye, J., and Ali, J. G. (2021). Cover crop soil legacies alter phytochemistry and resistance to fall armyworm (Lepidoptera: Noctuidae) in maize. Environ. Entomol. 50, 958-967. doi: 10.1093/ee/nvab047

De Groote, H., Kimenju, S. C., Munyua, B., Palmas, S., Kassie, M., and Bruce, A. (2020). Spread and impact of fall armyworm (Spodoptera frugiperda JE Smith) 
in maize production areas of Kenya. Agric. Ecosyst. Environ. 292:106804. doi: 10.1016/j.agee.2019.106804

de Mendiburu, F. (2014). agricolae: Statistical Procedures for Agricultural Research. $R$ Package Version 1.2-1.

de Vries, J., Evers, J. B., Dicke, M., and Poelman, E. H. (2019). Ecological interactions shape the adaptive value of plant defence: herbivore attack versus competition for light. Funct. Ecol. 33, 129-138. doi: 10.1111/1365-2435.13234

Early, R., González-Moreno, P., Murphy, S. T., and Day, R. (2018). Forecasting the global extent of invasion of the cereal pest Spodoptera frugiperda, the fall armyworm. NeoBiota 40, 25-50. doi: 10.3897/neobiota.40.28165

Endara, M., and Coley, P. D. (2011). The resource availability hypothesis revisited: a meta-analysis. Funct. Ecol. 25, 389-398.

Fescemyer, H. W., Sandoya, G. V., Gill, T. A., Ozkan, S., Marden, J. H., and Luthe, D. S. (2013). Maize toxin degrades peritrophic matrix proteins and stimulates compensatory transcriptome responses in fall armyworm midgut. Insect Biochem. Mol. Biol. 43, 280-291. doi: 10.1016/j.ibmb.2012.12.008

Gershenzon, J. (1994). Metabolic costs of terpenoid accumulation in higher plants. J. Chem. Ecol. 20, 1281-1328. doi: 10.1007/BF02059810

Gill, T. A., Sandoya, G., and Williams, P. (2011). Belowground resistance to western corn rootworm in lepidopteran-resistant maize genotypes. J. Econ. Entomol. 716, 299-307. doi: 10.1603/EC10117

Guo, Q., Major, I. T., and Howe, G. A. (2018). Resolution of growth - defense conflict : mechanistic insights from jasmonate signaling. Curr. Opin. Plant Biol. 44, 72-81. doi: 10.1016/j.pbi.2018.02.009

Hahn, P. G., and Maron, J. L. (2016). A framework for predicting intraspecific variation in plant defense. Trends Ecol. Evol. 31, 646-656. doi: 10.1016/j.tree. 2016.05.007

Hale, B. K., Herms, D. A., Hansen, R. C., Clausen, T. P., and Arnold, D. (2005). Effects of drought stress and nutrient availability on dry matter allocation, phenolic glycosides, and rapid induced resistance of poplar to two lymantriid defoliators. J. Chem. Ecol. 31, 2601-2620. doi: 10.1007/s10886-005-7616-8

Hambäck, P. A., and Beckerman, A. P. (2003). Herbivory and plant resource competition: a review of two interaction interactions. Oikos 101, 26-37. doi: 10.1034/j.1600-0706.2003.12568.x

Han, Y., Taylor, E. B., and Luthe, D. (2021). Maize endochitinase expression in response to fall armyworm herbivory. J. Chem. Ecol. 47, 689-706. doi: 10.1007/ s10886-021-01284-9

Hay, M. E., Kappel, Q. E., and Fenical, W. (1994). Synergisms in plant defenses against herbivores: interactions of chemistry, calcification, and plant-quality. Ecology 75, 1714-1726. doi: 10.2307/1939631

Herms, D. A., and Mattson, W. J. (1992). The dilemma of plants: to grow or defend. Q. Rev. Biol. 67, 283-335.

Karban, R. (2011). The ecology and evolution of induced resistance against herbivores. Funct. Ecol. 25, 339-347. doi: 10.1111/j.1365-2435.2010.01 789.x

Lavoie, B., and Oberhauser, K. S. (2004). Compensatory feeding in Danaus plexippus (Lepidoptera: Nymphalidae) in response to variation in host plant quality. Environ. Entomol. 33, 1062-1069. doi: 10.1603/0046-225X-33.4.1062

Livak, K. J., and Schmittgen, T. D. (2001). Analysis of relative gene expression data using real-time quantitative PCR and the 2- $\Delta \Delta$ CT method. Methods 25 , 402-408. doi: 10.1006/meth.2001.1262

Lou, Y., and Baldwin, I. T. (2004). Nitrogen supply influences herbivoreinduced direct and indirect defenses and transcriptional responses in Nicotiana attenuata. Plant Physiol. 135, 496-506. doi: 10.1104/pp.104.04 0360

Louis, J., Basu, S., Varsani, S., Castano-duque, L., Jiang, V., Williams, W. P., et al. (2015). Ethylene contributes to maize insect resistancel -mediated maize defense against the phloem sap-sucking. Plant Physiol. 169, 313-324. doi: 10. 1104/pp.15.00958

Mason, C. J., Ray, S., Shikano, I., Peiffer, M., Jones, A. G., Luthe, D. S., et al. (2019). Plant defenses interact with insect enteric bacteria by initiating a leaky gut syndrome. Proc. Natl. Acad. Sci. U. S. A. 116, 15991-15996. doi: 10.1073/pnas. 1908748116

Mertens, D., Boege, K., Kessler, A., Koricheva, J., Thaler, J. S., Whiteman, N. K., et al. (2021). Predictability of biotic stress structures plant defence evolution. Trends Ecol. Evol. 36, 444-456. doi: 10.1016/j.tree.2020.12.009

Mitchell, C., Brennan, R. M., Graham, J., and Karley, A. J. (2016). Plant defense against herbivorous pests: exploiting resistance and tolerance traits for sustainable crop protection. Front. Plant Sci. 7:1132. doi: 10.3389/fpls.2016. 01132

Mohan, S., Ma, P. W., Pechan, T., Bassford, E. R., Williams, W. P., and Luthe, D. S. (2006). Degradation of the $S$. frugiperda peritrophic matrix by an inducible maize cysteine protease. J. Insect Physiol. 52, 21-28. doi: 10.1016/j.jinsphys. 2005.08.011

Moles, A. T., Peco, B., Wallis, I. R., Foley, W. J., Poore, A. G. B., Seabloom, E. W., et al. (2013). Correlations between physical and chemical defences in plants: tradeoffs, syndromes, or just many different ways to skin a herbivorous cat? New Phytol. 198, 252-263. doi: 10.1111/nph.12116

Montezano, D. G., Specht, A., Sosa-Gómez, D. R., Roque-Specht, V. F., Sousa-Silva, J. C., Paula-Moraes, S. V., et al. (2018). Host plants of Spodoptera frugiperda (Lepidoptera: Noctuidae) in the Americas. Afr. Entomol. 26, 286-300. doi: 10.4001/003.026.0286

Morris, S. W., Vernooij, B., Titatarn, S., Starrett, M., Thomas, S., Wiltse, C. C., et al. (1998). Induced resistance responses in maize. Molecular Plant Microbe Interact. 11, 643-658. doi: 10.1094/MPMI.1998.11.7.643

Murrell, E. G., Ray, S., Lemmon, M. E., Luthe, D. S., and Kaye, J. P. (2019). Cover crop species affect mycorrhizae-mediated nutrient uptake and pest resistance in maize. Renew. Agric. Food Syst. 35, 467-474. doi: 10.1017/S1742170519000061

Mustafa, G., Randoux, B., Tisserant, B., Fontaine, J., Magnin-Robert, M., LounèsHadj Sahraoui, A., et al. (2016). Phosphorus supply, arbuscular mycorrhizal fungal species, and plant genotype impact on the protective efficacy of mycorrhizal inoculation against wheat powdery mildew. Mycorrhiza 26, 685697. doi: 10.1007/s00572-016-0698-z

Pechan, T., Cohen, A., Williams, W. P., and Luthe, D. S. (2002). Insect feeding mobilizes a unique plant defense protease that disrupts the peritrophic matrix of caterpillars. Proc. Natl. Acad. Sci. U. S. A. 99, 13319-13323. doi: 10.1073/pnas. 202224899

Pechan, T., Ye, L., Chang, Y., Mitra, A., Lin, L., Davis, F. M., et al. (2000). A unique $33-\mathrm{kD}$ cysteine proteinase accumulates in response to larval feeding in maize genotypes resistant to fall armyworm and other Lepidoptera. Plant Cell 12, 1031-1040. doi: 10.1105/tpc. 12.7.1031

Phillips, M. A., and Croteau, R. B. (1999). Resin-based defenses in conifers. Trends Plant Sci. 4, 184-190. doi: 10.1016/s1360-1385(99)01401-6

Prudic, K. L., Oliver, J. C., and Bowers, M. D. (2005). Soil nutrient effects on oviposition preference, larval performance, and chemical defense of a specialist insect herbivore. Oecologia 143, 578-587. doi: 10.1007/s00442-005-0008-5

R Core Team (2020). R: A Language and Environment for Statistical Computing. Vienna: R Foundation for Statistical Computing.

Rashid, M. M., Ahmed, N., Jahan, M., Islam, K. S., Nansen, C., Willers, J. L., et al. (2017). Higher fertilizer inputs increase fitness traits of brown planthopper in rice. Sci. Rep. 7:4719. doi: 10.1038/s41598-017-05023-7

Ray, S., Alves, P. C., Ahmad, I., Gaffoor, I., Acevedo, F. E., Peiffer, M., et al. (2016). Turnabout is fair play: herbivory-induced plant chitinases excreted in fall armyworm frass suppress herbivore defenses in maize. Plant Physiol. 171, 694-706. doi: 10.1104/pp.15.01854

Raza, A., Charagh, S., Zahid, Z., Mubarik, M. S., Javed, R., Siddiqui, M. H., et al. (2021). Jasmonic acid: a key frontier in conferring abiotic stress tolerance in plants. Plant Cell Rep. 40, 1513-1541. doi: 10.1007/s00299-020-02614-z

RStudio Team (2015). RStudio: Integrated Development for R. Boston: RStudio, Inc. Schmelz, E. A., Alborn, H. T., Engelberth, J., and Tumlinson, J. H. (2003). Nitrogen deficiency increases volicitin-induced volatile emission, jasmonic acid accumulation, and ethylene sensitivity in maize. Plant Physiol. 133, 295-306. doi: 10.1104/pp.103.024174

Sethi, A., McAuslane, H. J., Alborn, H. T., Nagata, R. T., and Nuessly, G. S. (2008). Romaine lettuce latex deters feeding of banded cucumber beetle: a vehicle for deployment of biochemical defenses. Entomol. Exp. Appl. 128, 410-420. doi: 10.1111/j.1570-7458.2008.00732.x

Shivaji, R., Camas, A., Ankala, A., Engelberth, J., Tumlinson, J., Williams, W., et al. (2010). Plants on constant alert: elevated levels of jasmonic acid and jasmonateinduced transcripts in caterpillar-resistant maize. J. Chem. Ecol. 36, 179-191. doi: 10.1007/s10886-010-9752-z

Singh, G., Waltz, F. C. Jr., and Joseph, S. V. (2021). Potassium and nitrogen impacts on survival and development of fall armyworm (Lepidoptera: Noctuidae). J. Entomol. Sci. 56, 411-423. doi: 10.18474/JES20-58

Stamp, N. (2003). Out of the quagmire of plant defense hypotheses. Q. Rev. Biol. 78, 23-55. doi: $10.1086 / 367580$ 
Steppuhn, A., and Baldwin, I. T. (2008). "Induced defenses and the cost-benefit paradigm," in Induced Plant Resistance to Herbivory, ed. A. Haller (Berlin: Springer), 61-88. doi: 10.1007/978-1-4020-8182-8

Stout, M. J., Brovont, R. A., and Duffey, S. S. (1998). Effect of nitrogen avilability on expression of constitutive and inducible chemical defenses in tomato, Lycopersicon esculentum. J. Chem. Ecol. 24, 945-963. doi: 10.1023/A: 1022350100718

Tian, T., Tooker, J., Peiffer, M., Chung, S. H., and Felton, G. W. (2012). Role of trichomes in defense against herbivores : comparison of herbivore response to woolly and hairless trichome. Planta 236, 1053-1066. doi: 10.1007/s00425-0121651-9

Tzin, V., Hojo, Y., Strickler, S. R., Bartsch, L. J., Archer, C. M., Ahern, K. R., et al. (2017). Rapid defense responses in maize leaves induced by Spodoptera exigua caterpillar feeding. J. Exp. Bot. 68, 4709-4723. doi: 10.1093/jxb/erx274

Walls, R., Appel, H., Cipollini, M., and Schultz, J. (2005). Fertility, root reserves and the cost of inducible defenses in the perennial plant Solanum carolinense. J. Chem. Ecol. 31, 2263-2288. doi: 10.1007/s10886-005-7101-4

Wetzel, W. C., Kharouba, H. M., Robinson, M., Holyoak, M., and Karban, R. (2016). Variability in plant nutrients reduces insect herbivore performance. Nature 539, 425-427. doi: 10.1038/nature20140

Williams, W. P., Davis, F. M., and Windham, G. L. (1990). Registration of Mp708 Germplasm Line of Maize. Crop Sci. 30:757. doi: 10.2135/cropsci2002.3120

Wilson, J. K., Ruiz, L., Duarte, J., and Davidowitz, G. (2019). The nutritional landscape of host plants for a specialist insect herbivore. Ecol. Evol. 9, 1310413113. doi: 10.1002/ece3.5730

Wiseman, B. R., Leuck, D., and McMillian, W. W. (1973). Effects of fertilizers on resistance of antigua corn to fall armyworm and corn earworm. Florida Entomol. 56, 1-7. doi: 10.2307/3493653
Zhu-Salzman, K., and Zeng, R. (2015). Insect response to plant defensive protease inhibitors. Annu. Rev. Entomol. 60, 233-252. doi: 10.1146/annurev-ento010814-020816

Züst, T., and Agrawal, A. A. (2017). Trade-offs between plant growth and defense against insect herbivory: an emerging mechanistic synthesis. Ann. Rev. Plant Biol. 68, 513-534. doi: 10.1146/annurev-arplant-042916-04 0856

Author Disclaimer: All opinions expressed in this manuscript are the author's and do not necessarily reflect the policies and views of USDA, DOE or ORAU/ORISE.

Conflict of Interest: The authors declare that the research was conducted in the absence of any commercial or financial relationships that could be construed as a potential conflict of interest.

Publisher's Note: All claims expressed in this article are solely those of the authors and do not necessarily represent those of their affiliated organizations, or those of the publisher, the editors and the reviewers. Any product that may be evaluated in this article, or claim that may be made by its manufacturer, is not guaranteed or endorsed by the publisher.

Copyright (C) 2022 Mason, Ray, Davidson-Lowe, Ali, Luthe and Felton. This is an open-access article distributed under the terms of the Creative Commons Attribution License (CC BY). The use, distribution or reproduction in other forums is permitted, provided the original author(s) and the copyright owner(s) are credited and that the original publication in this journal is cited, in accordance with accepted academic practice. No use, distribution or reproduction is permitted which does not comply with these terms. 